\title{
PROPUESTA METODOLÓGICA BASADA EN INDICADORES PARA LA VALORACIÓN DEL POTENCIAL TURÍSTICO DEL PAISAJE EN ÁREAS RURALES: EL CASO DEL MUNICIPIO DE ATLAUTLA (MÉXICO) ${ }^{1}$
}

\author{
Alberto Méndez-Méndez, \\ Universidad Nacional Autónoma de México \\ Manuel Antonio Serrano de la Cruz Santos-Olmo** \\ Universidad de Castilla-La Mancha \\ Eduardo Salinas Chávez**** \\ Universidad Federal de Mato Grosso do Sul \\ Arturo García-Romero***** \\ Universidad Nacional Autónoma de México
}

\section{RESUMEN}

La consideración del paisaje como una herramienta para la planificación del desarrollo turístico es cada vez más común en las zonas rurales de todo el mundo. En este artículo se describe un nuevo método para evaluar el potencial turístico del paisaje en las zonas rurales que aún no se han incorporado al turismo, pero donde se conoce la existencia de un amplio capital natural y cultural. El método se basa en el diseño y uso de indicadores para evaluar la calidad de dos recursos complementarios del potencial turístico: los tipos de paisaje y los

Fecha de recepción: 1 de septiembre de 2017.

Fecha de aceptación: 12 de marzo de 2018.

* Facultad de Estudios Superiores Zaragoza. Universidad Nacional Autónoma de México. Batalla 5 de Mayo SN, Iztapalapa, Ejército Oriente.09230 Ciudad de México.E-mail: albmen@unam.mx

** Departamento de Geografía y Ordenación del Territorio. Universidad de Castilla-La Mancha. Avda. Camilo José Cela s/n. 13071 Ciudad Real (España).E-mail: Manuel.SerranoCruz@uclm.es

**** Universidad Federal de Mato Grosso do Sul, Campus Tres Lagoas, MS, Brasil.E-mail: esalinasc@yahoo.com

**** Departamento de Geografía Física. Instituto de Geografía. Universidad Nacional Autónoma de México. Investigación Científica. Ciudad Universitaria. 04510 Ciudad de México. E-mail: agromero@igg.unam.mx

1 Este artículo es resultado de la investigación realizada con financiamiento del Proyecto IN301218 y el Programa Nacional de Becas para Estudios de Posgrado del Consejo Nacional de Ciencia y Tecnología de México que apoyó al primer autor. 
atractivos turísticos. La aplicación del método en un municipio del centro de México permitió determinar el potencial turístico de los tipos de paisaje a partir de su calidad intrínseca, más la consideración de la calidad de los atractivos turísticos que albergan en su interior. Los resultados destacan la importancia de la relación paisaje-turismo, considerada en varias escalas de análisis, como base para la planificación y gestión de proyectos de turismo sostenible.

Palabras clave: Calidad del paisaje, atractivo turístico, potencial turístico del paisaje, indicador ambiental, planificación turística, turismo rural.

\title{
Methodological proposal based on indicators for the assessment of the landscape
} tourist potential in rural areas: the case of the municipality of Atlautla (Mexico)

\begin{abstract}
Including the landscape as a tool for tourism development planning is becoming more common in rural areas around the world. This paper describes a new method for evaluating the tourism potential of landscape in rural areas that are not incorporated to tourism yet, but where the existence of a broad natural and cultural capital is known. The method here proposed is based on the design and use of indicators to evaluate the quality of two complementary resources of tourism potential: types of landscape and tourist attractions. The application of the method in a municipality of central Mexico allowed determining the landscape type's tourist potential based on its intrinsic quality plus the consideration of the quality of the tourist attractions that shelter in its interior. The results highlight the importance of the landscape-tourism relationship, considered at various scales of analysis, as the basis for planning sustainable tourism projects.
\end{abstract}

Keywords: Landscape quality, tourist attraction, tourism potential of landscape, environmental indicator, tourism planning, rural tourism.

\section{INTRODUCCIÓN}

El paisaje ha sido considerado como parte del potencial turístico del territorio desde los años sesenta del siglo XX, cuando se formularon los primeros métodos para evaluar el potencial de los diversos recursos disponibles (Dosso, 2011). Desde entonces, el paisaje se ha posicionado, cada vez más, como elemento básico para la identificación y valoración de la calidad de los recursos turísticos, considerados ellos como uno de los principales componentes de la compleja estructura del sistema turístico (Alcalá y López, 2017). En la mayoría de los casos, el paisaje se aborda desde un punto de vista visual y estético, proporcionando interpretaciones interesantes del enfoque afectivo o perceptivo-psicológico evidente para los turistas (Nogué, 1992; Zuluaga, 2006; Knudsen et al., 2013; Kalivoda et al., 2014). Más recientemente, la tendencia hacia un turismo cada vez más informado, justo y sostenible ha permitido considerar el paisaje como una herramienta útil de alto valor explicativo, que añade al valor estético otros como el ecológico, educativo, interpretativo, histórico, etc., que apuestan por la autenticidad y singularidad frente a la estandarización de los lugares (Tarroja, 2004; Zuluaga, 2006; Meng y Uysal, 2008; Picazo, 2012; Lane y Kastenholtz, 2015; Cebrián y García, 2016; Jerez y Serrano de la Cruz, 2016). 
Una de las herramientas propuestas para la adecuada planificación de los proyectos es la evaluación del potencial turístico de los paisajes (Zuluaga, 2006; Dosso, 2011), que se define como el conjunto de condiciones naturales y culturales que hacen que el paisaje sea adecuado para el desarrollo de las actividades turísticas (Picazo, 2012; Santos-Pavón, et al., 2016), lo cual lo hace no sólo un elemento complementario, sino un recurso y apoyo básico para el turismo y para la creación del valor de los lugares (Aitchison et al., 2001; Nogué et al., 2009; Picazo, 2012; Knudsen et al., 2008, 2013; Wlodarczyk, 2009).

Gran parte de los proyectos de desarrollo turístico en la actualidad se encuentran en zonas rurales, donde existen recursos naturales y/o culturales a menudo subvalorados, pero que pueden ser explotados como atractivos turísticos. En muchos casos, el turismo ha sido incluso propuesto como una alternativa o un complemento al desarrollo local de las zonas rurales tradicionales. Sin embargo, siendo una actividad compleja, la necesidad de esquemas de planificación que garanticen la sostenibilidad de tales proyectos turísticos es cada vez más urgente, particularmente en las zonas rurales de los países en desarrollo.

Por otro lado, desde un punto de vista operativo, vale la pena destacar el uso de indicadores para evaluar de manera precisa y sistemática el potencial turístico del paisaje (Serrano, 2008; Nogué et al., 2009). El diseño, la validación y la aplicación de indicadores a casos específicos proporcionan valores de referencia que son útiles para el diagnóstico y, eventualmente, la intervención en el paisaje. La aplicación de indicadores sigue estando poco desarrollada, pero se pueden señalar algunas experiencias interesantes en la evaluación de la identidad, la calidad o el atractivo visual del paisaje (Carneiro et al., 2015; Serrano, 2008; Salinas, et al., 2008). En general, la utilidad de los indicadores radica en que son suficientemente abiertos para acomodar una amplia gama de casos, pero lo suficientemente puntuales para precisar las evaluaciones (Nogué et al., 2009; OECD, 2003).

A diferencia de los estudios realizados durante la fase operacional, o a posteriori, del turismo, que permiten alcanzar los objetivos relacionados con el desempeño económico (Mearns, 2012) o el impacto ambiental (impactos en el capital del turismo) de los turistas (Mikulec y Antoušková, 2011), en este caso se presenta un método de diagnóstico basado en indicadores para identificar y evaluar el potencial turístico del paisaje y la viabilidad de proyectos en nuevas áreas, todavía no incorporadas a este tipo de actividad, pero donde se presume la existencia de un amplio capital natural y cultural, favoreciendo así los efectos positivos de la actividad turística (Tarroja, 2004; Barbini, 2005).

El ejercicio metodológico se desarrolló en una comunidad rural de bosques y montañas del centro de México, caracterizada por una gran riqueza natural y cultural, pero con evidentes condiciones de pobreza y complejas perspectivas de desarrollo. La propuesta es aplicable a otras comunidades rurales en las que la planificación de proyectos turísticos permita ofrecer una oportunidad de desarrollo, amparada en el diagnóstico integral del potencial turístico del paisaje, capaz de garantizar su viabilidad, la equidad de los beneficios y la adecuada valoración de los recursos paisajísticos.

En el proceso metodológico seguido, se partió de la identificación de los tipos de paisaje en el área de estudio y del inventario de los atractivos turísticos en cada tipo de paisaje. Se definió un conjunto de indicadores objetivos y mesurables para describir las características escénicas, ambientales y culturales del paisaje, como la base para determinar la calidad de los tipos de paisaje y de los atractivos turísticos locales. Por último, se 
realizó un ejercicio de integración y unificación, que ha permitido la construcción de una clasificación paisajística en base a un potencial turístico alto, medio o bajo, susceptible de ser utilizado como propuesta para el diseño e implementación de esquemas de planificación y gestión para el desarrollo del turismo en el área de estudio.

\section{2. ÁREA DE ESTUDIO}

La investigación se llevó a cabo en el municipio de Atlautla (167,56km²; 27.663 hab.), localizado en la vertiente occidental del volcán Popocatépetl, a $75 \mathrm{~km}$ al SE de la Ciudad de México (Figura 1). El desnivel altitudinal, de más de 3000 m ( 2200-5426 msnm), favorece la diversidad ambiental, incluyendo cinco pisos de vegetación - desde los encinares (Quercus spp.) de la base, a los bosques mixtos (encinos y coníferas), bosques de coníferas (pinos y abetos), pinares de alta montaña (Pinus hartwegii) y pastizales de alta montaña (INEGI, 1983, 2009) -, además de una amplia diversidad de formas tradicionales de uso del suelo que se concentran en la base de la vertiente.

Aunque el municipio se encuentra próximo a un corredor turístico-económico de importante afluencia turística, básicamente interesada en actividades de recreación y esparcimiento en espacios forestales y de alta montaña, esta área no ha logrado aún posicionarse de manera sobresaliente en el sistema turístico regional. El patrón de usos del suelo incluye: bosques $(45,2 \%)$, cultivos $(30,3 \%)$, pastizales $(9,6 \%)$, áreas sin vegetación $(9,3 \%)$ y áreas urbanas $(5,6 \%)$ (INEGI, 2009), reflejando una economía basada en la agricultura (principalmente de maíz, frijol, hortalizas y frutales), sumida en diversos conflictos relacionados con la propiedad de la tierra, la falta de infraestructuras y de un comercio organizado, y la pobreza y emigración de la población joven.

\section{MATERIALES Y MÉTODOS}

El método desarrollado incluye cuatro etapas agrupadas en dos fases principales. La primera fase comprende la identificación, caracterización y cartografía de los paisajes y los atractivos turísticos; y la segunda fase comprende la valoración de la calidad turística del paisaje y sus atractivos, y la integración y síntesis para determinar el potencial turístico del paisaje.

\subsection{Identificación, caracterización y cartografía de los paisajes y los atractivos turísticos}

Se elaboró un mapa de paisajes a partir de un criterio taxonómico y jerárquico que incluye dos niveles de análisis: tipo de paisaje y unidad de paisaje. Cada nivel taxonómico tiene una homogeneidad relativa de los elementos que lo componen y que lo diferencian del otro, y que se define por el carácter de la manifestación de los procesos ambientales que en ellos se llevan a cabo y sus interrelaciones (Muñoz, 1998; García-Romero, 2002; Mateo, 2008).

a) Tipo de paisaje: Patrón paisajístico (cientos a decenas de $\mathrm{km}^{2}$ ) coincidente, en este caso, con la combinación de una unidad superior de relieve y una variante del clima 
regional. De estos componentes mayores depende la distribución de los elementos orográficos, altitudinales, de orientación y litológicos, así como ciertos parámetros climáticos (en particular térmicos y pluviométricos), que en conjunto inciden en las características del patrón paisajístico contenido en su interior.

b) Unidad de paisaje: Unidad paisajística (miles a cientos de $\mathrm{m}^{2}$ ) definida por la combinación de componentes abióticos -hidrología y relieve- que influyen sobre otros componentes de carácter biótico y cultural -suelo, vegetación, uso del suelo-, altamente inestables, dependientes y dinámicos. Al interior de un tipo de paisaje, sus unidades de paisaje comparten un mismo ambiente climático y morfoestructural.

El mapa de paisajes se elaboró - en SIG's ILWIS ver. 3.4 y ArcMap 10.1 - sobre la base de la imagen satelital del Servicio de Imágenes del Planeta Google Earth ${ }^{\mathrm{TM}}$ de 2014, y de la cartografía topográfica y de uso de suelo y vegetación escala 1:50.000 de la institución cartográfica en el país (INEGI, 1983, 1996). La información fue validada y actualizada en campo.

En el caso de los atractivos turísticos, estos fueron inventariados durante una serie de visitas programadas al área de estudio según las recomendaciones de varios informantes clave del municipio. Las características de cada atractivo se registraron en una hoja de datos de campo que contenía los indicadores de calidad propuestos. Las hojas de datos se cumplimentaron en el campo siguiendo el método Delphi (Gordon, 1994, Mateos, 2016), y los resultados se cartografiaron y se introdujeron en la base de datos asociada para un análisis posterior.

\subsection{Valoración de la calidad turística del paisaje y sus atractivos turísticos, y síntesis del potencial turístico del paisaje}

La calidad turística de los tipos paisajísticos y sus atractivos se evaluó mediante indicadores relacionados con tres criterios principales:

A) Escénico: Considerado para evaluar la percepción de la belleza del paisaje. Las características analizadas son la cantidad y calidad de los componentes que definen el patrón del paisaje local. El contraste visual entre las formas, los colores, la perspectiva resultante y la presencia de componentes excepcionales de alto valor. Este criterio considera tanto el valor escénico del sitio en sí como el valor resultante de la amplitud panorámica, que permite vistas de larga distancia sin obstáculos desde sitios puntuales o sobre trayectos longitudinales.

B) Ambiental: Considerado para evaluar la estabilidad geomorfológica, el grado de conservación de los componentes bióticos y abióticos naturales, y la productividad, sostenibilidad, accesibilidad e instalaciones asociadas a los componentes culturales del paisaje. Esto implica la importancia del patrimonio material (por ejemplo, la infraestructura) e intangible (cultural e histórico-espiritual) asociado con el sitio, así como la originalidad e importancia de la relación entre los componentes del paisaje y el peso de algunos de ellos para la administración o protección de los demás. 
C) Interpretativo: Considerado para evaluar las características didácticas y de ejemplaridad del sitio como representantes de la singularidad de los componentes del paisaje y su importancia para facilitar las acciones educativas, o despertar interés en los estudios académicos, para la comprensión del paisaje. Las características importantes del sitio, a este respecto, son el alcance relacionado tanto con la calidad de los componentes locales y la configuración general del paisaje.

Para determinar la calidad de los tipos de paisaje se consideraron, a su vez, tres indicadores, uno para cada uno de los criterios seleccionados: a) diversidad del paisaje, como indicador del valor escénico del paisaje; b) fragmentación espacial, como indicador de estabilidad ambiental; y c) representatividad/singularidad, como indicador del valor interpretativo del paisaje (Tabla 1).

Los atractivos turísticos se evaluaron en función de tres componentes básicos del paisaje: geomorfológicos, biológicos y culturales. A partir de este esquema, se diseñaron los indicadores para cada criterio con respecto a cada componente del paisaje (Tabla 2). Es importante destacar que los indicadores propuestos fueron identificados con el apoyo de informantes locales involucrados en el turismo.

\section{Tabla 1}

\section{INDICADORES UTILIZADOS PARA EVALUAR LA CALIDAD TURÍSTICA DE LOS TIPOS DE PAISAJE}

\begin{tabular}{|c|c|c|}
\hline Criterio/Indicador & Descripción & Medición \\
\hline $\begin{array}{l}\text { Diversidad } \\
\text { visual del patrón } \\
\text { paisajístico }\end{array}$ & $\begin{array}{l}\text { Variedad de las unidades } \\
\text { de paisaje (combinaciones } \\
\text { de geoforma, tipo de } \\
\text { vegetación y uso del suelo } \\
\text { específico), contenida en } \\
\text { el interior de cada tipo de } \\
\text { paisaje. }\end{array}$ & $\begin{array}{l}\text { Índice de Diversidad de Simpson }\left(\mathrm{Si}_{D}\right) \\
\text { (Simpson, 1949; Peet, 1974): } \\
\qquad S i_{D}=1-\sum_{i=1}^{S} p_{i}{ }^{2} \\
\text { Donde } P i=\text { Proporción de la superficie del } \\
\text { tipo de paisaje ocupada por el paisaje } i ; S= \\
\text { Número de unidades de paisaje. }\end{array}$ \\
\hline $\begin{array}{l}\text { Fragmentación } \\
\text { espacial del patrón } \\
\text { paisajístico }\end{array}$ & $\begin{array}{l}\text { Cantidad de fragmentos, } \\
\text { en relación a la superficie } \\
\text { total del tipo de paisaje. } \\
\text { Un paisaje fragmentado se } \\
\text { relaciona con alteraciones } \\
\text { y es más sensible a } \\
\text { procesos de perturbación. }\end{array}$ & $\begin{array}{l}\text { Índice de división S (SPLI) (Jaeger, 2000): } \\
\qquad S P L I=\frac{A_{t}^{2}}{\sum_{n=1}^{n} A_{i}^{2}} \\
\text { Donde } \mathrm{n}=\text { número de fragmentos de paisaje; } \\
\text { Ai }=\text { tamaño de los } \mathrm{n} \text { fragmentos de paisaje } \\
(\mathrm{i}=1, \ldots, \mathrm{n}) ; \mathrm{At}=\text { Área total del tipo de paisaje. }\end{array}$ \\
\hline $\begin{array}{l}\text { Representatividad } \\
\text { y/o singularidad del } \\
\text { patrón paisajístico }\end{array}$ & $\begin{array}{l}\text { Características didácticas } \\
\text { y de ejemplaridad del } \\
\text { patrón paisajístico } \\
\text { como representativo de } \\
\text { elementos históricos, }\end{array}$ & $\begin{array}{l}\text { Índice de singularidad/representatividad }\left(I_{S / R}\right) \text {, } \\
\text { es un índice } a d \text { hoc que combina elementos } \\
\text { de los tres componentes básicos del paisaje: } \\
\text { uso del suelo, relieve y vegetación (García- } \\
\text { Romero, 2002). Las variables utilizadas }\end{array}$ \\
\hline
\end{tabular}




\begin{tabular}{|c|c|c|}
\hline Criterio/Indicador & Descripción & Medición \\
\hline $\begin{array}{l}\text { Valor Interpretativo/ } \\
\text { Representatividad } \\
\text { y/o singularidad del } \\
\text { patrón paisajístico }\end{array}$ & $\begin{array}{l}\text { artísticos o de uso del } \\
\text { suelo, y de su relación } \\
\text { armoniosa con aspectos } \\
\text { relativos al origen y } \\
\text { procesos biofísicos propios } \\
\text { de la región, tales que le } \\
\text { confieren trascendencia e } \\
\text { identidad cultural. }\end{array}$ & $\begin{array}{l}\text { fueron: Productividad del sistema agropecuario } \\
\text { o forestal (Ps) (Torres, 2003; Nikodemus et } \\
\text { al., 2005), Estabilidad del relieve (Er) (Smith } \\
\text { et al., 2008) y Estado de conservación de la } \\
\text { vegetación) (Cv) (Powell et al., 2000). } \\
\qquad I_{R / S}=P s+E r+C v \\
\text { Donde } P S, E r \text { y } C v \text { son, respectivamente, las } \\
\text { proporciones de la superficie con usos del } \\
\text { suelo activos, relieve estable y vegetación } \\
\text { conservada en cada tipo. }\end{array}$ \\
\hline
\end{tabular}

Tabla 2

INDICADORES PARA EVALUAR LA CALIDAD DE LOS ATRACTIVOS TURÍSTICOS. SE INDICAN LOS COMPONENTES DEL PAISAJE Y LOS CRITERIOS DE VALORACIÓN CONSIDERADOS

\begin{tabular}{|c|c|c|c|c|}
\hline & \multicolumn{3}{|c|}{ Indicadores de calidad por componente del paisaje } \\
\hline & & Biótico & Geomorfológico & Cultural \\
\hline \multirow[t]{2}{*}{ 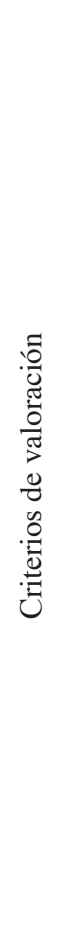 } & Ambiental & $\begin{array}{l}\text { - Estado de } \\
\text { conservación } \\
\text { - Cercanía a } \\
\text { Áreas Naturales } \\
\text { Protegidas } \\
\text { - Servicios } \\
\text { ambientales }\end{array}$ & $\begin{array}{l}\text { - Calidad escénica } \\
\text { intrínseca } \\
\text { - Desnivel topográfico } \\
\text { - Presencia de cuerpos } \\
\text { de agua } \\
\text { - Amplitud panorámica } \\
\text { - Variedad paisajística } \\
\text { panorámica } \\
\text { - Longitud del tramo con } \\
\text { panorámicas }\end{array}$ & $\begin{array}{l}\text { - Trascendencia religiosa } \\
\text { o mística } \\
\text { - Trascendencia histórica } \\
\text { - Trascendencia artística y } \\
\text { cultural } \\
\text { - Productos económicos } \\
\text { - Nivel de alcance del } \\
\text { atractivo } \\
\text { - Equipamiento } \\
\text { - Tipo de acceso } \\
\text { - Distancia al sitio }\end{array}$ \\
\hline & Interpretativo & $\begin{array}{l}\text { - Representatividad } \\
\text { - Singularidad }\end{array}$ & $\begin{array}{l}\text { - Representatividad } \\
\text { - Singularidad }\end{array}$ & $\begin{array}{l}\text { - Representatividad } \\
\text { - Singularidad }\end{array}$ \\
\hline
\end{tabular}


La calidad de cada indicador se calificó usando una escala ordinal de 5 valores $(0$, $0.25,0.5,0.75$ y 1$)$, donde 1 indica la calidad más alta que se puede lograr en un sitio dado. Con estas calificaciones se determinó la calidad de cada atracción turística (Cat) mediante la fórmula siguiente:

$$
C a t=x(V b)+y(V g)+z(V c)
$$

Donde (Cat) es la calidad del atractivo turístico; $(V b)$ es la suma de las puntuaciones para los indicadores del componente biótico; $(V g)$ es la suma de las puntuaciones para los indicadores del componente geomorfológico; $(V c)$ es la suma de puntajes para los indicadores del componente cultural; $x, y, z$ son factores de ponderación necesarios para evitar la sobre o subestimación de los componentes. Estos factores de ponderación se obtuvieron utilizando la ecuación siguiente:

$$
\text { Factor }(y, y, z)=[(n b+n g+n c) * 0,333] / n(b, g, c)
$$

Dado que en este estudio se utilizaron 29 indicadores, 6 para el componente biótico $(n b), 11$ para el geomorfológico $(n g)$ y 12 para el cultural $(n c)$ (Tabla 2), la fórmula final para obtener Cat fue:

$$
C a t=1,61(V b)+0,88(V g)+0,81(V c)
$$

Por último, los atractivos turísticos se clasificaron en función de su valor Cat, en relación con su máximo valor posible $\left(C a t_{\max }=29\right)$, cuando todos los indicadores tienen la puntuación más alta (1). Así, se definieron tres categorías: Baja (inferior a 9,67), Media (entre 9,67 y 19,36) y Alta (entre 19,37 y 29).

El potencial turístico de los paisajes tiene una relación directa y proporcional con la calidad expresada por cada uno de los índices utilizados, sin embargo, al ser estos dimensionalmente independientes entre sí, se interpretan por separado, de tal manera que la calidad no es la suma simple de dichos valores, sino el resultado de su interpretación cualitativa y pragmática. En este sentido, con base en el significado y los umbrales propios de cada índice, se les asoció a una de las tres categorías establecidas (Baja, Media y Alta), que permite homogenizar los resultados y facilitar su interpretación. La calidad, expresada de esta manera, permite reconocer en qué medida contribuyen los indicadores utilizados en cada criterio de valoración (diversidad, fragmentación representatividad/singularidad). Posteriormente, se agregó el valor de calidad de los atractivos turísticos ( $\mathrm{Cat}$ ) existentes en cada tipo de paisaje. Así, la Cat actúa como un valor agregado de la calidad y como elemento de juicio para comparar la calidad turística de los paisajes. 


\section{RESULTADOS}

\subsection{Clasificación y valoración de los paisajes para el aprovechamiento turístico}

Se identificaron cuatro tipos de paisajes en el municipio de Atlautla, cada uno diferenciado principalmente por el sustrato geológico volcánico que, junto con algunos atributos morfológicos que afectan la variabilidad bioclimática de las laderas, producen estructuras paisajísticas estables a escala humana (Bertrand y Bertrand, 2006) (Tabla 3 y Figura 1). Los cuatro tipos de paisaje identificados son: 1) Piedemonte piroclástico con bosque mixto (PP); 2) Piedemonte lávico con bosque de encino, uso agrícola disperso y poblamiento concentrado en pueblos (PL); 3) Ladera media con bosque de coníferas y uso forestal (LM); y 4) Alta montaña con bosque de pino y pastizal alpino (AM).

Los patrones en los tipos de paisaje incluyen 40 unidades de paisaje diferentes, que son evidencia de la diversidad geoecológica de las laderas occidentales del volcán Popocatépetl. Los componentes naturales incluyen bosques de coníferas en las laderas, barrancos y domos volcánicos de las montañas altas, y laderas escarpadas típicas de la ladera media. Los bosques mixtos y de coníferas también están bien representados, especialmente en los barrancos, flujos de lava y en las colinas del piedemonte piroclástico y del piedemonte lávico. También destacan los paisajes alpinos de praderas y afloramientos rocosos en las laderas, barrancos superiores y domos volcánicos parcialmente cubiertos por arena volcánica reciente en la cumbre del volcán Popocatépetl (Tabla 3).

Las actividades agrícolas se realizan sobre vastas tierras de cultivo en las colinas del piedemonte piroclástico y en áreas más pequeñas en planicies de inundación, terrazas y valles de fondo plano. Paisajes naturales degradados y paisajes antrópicos que ocurren en áreas predominantemente agrícolas. Los primeros consisten principalmente en vegetación secundaria que cubre extensas áreas en la ladera media y las colinas y flujos de lava del piedemonte lávico. Los paisajes antropogénicos corresponden a asentamientos humanos en las zonas bajas de los piedemontes lávico y piroclástico (Tabla 3).

Sólo cinco unidades de paisaje (bosques de coníferas en laderas medias, tierras de cultivo en lomeríos piroclásticos, bosques mixtos en barrancos, arenas volcánicas en laderas altas y cultivos en domos y flujos de lava) cubren el 53\% del área de estudio y parecen concentradas principalmente en el piedemonte piroclástico y la alta montaña del volcán Popocatépetl.

La calidad de los tipos de paisaje proviene del conjunto de unidades que definen su patrón paisajístico. En este sentido, los valores obtenidos muestran que la diversidad de patrones paisajísticos es ligeramente superior en la alta montaña y en el piedemonte lávico, que en el piedemonte piroclástico y la ladera media (Tabla 4). El índice de diversidad de Simpson tiene en cuenta tanto la variedad de unidades de paisaje (riqueza) como la relación de dominancia entre ellas, mostrando así el predominio de los bosques de coníferas en laderas medias y la mayor uniformidad entre las superficies cubiertas por las unidades de paisaje en la alta montaña.

Por otro lado, la fragmentación (considerada aquí como un factor ambientalmente adverso), se interpretó a partir del índice de división (Tabla 1), que es un indicador indirecto de la estabilidad y la resiliencia del patrón de paisaje. La fragmentación es ligera- 


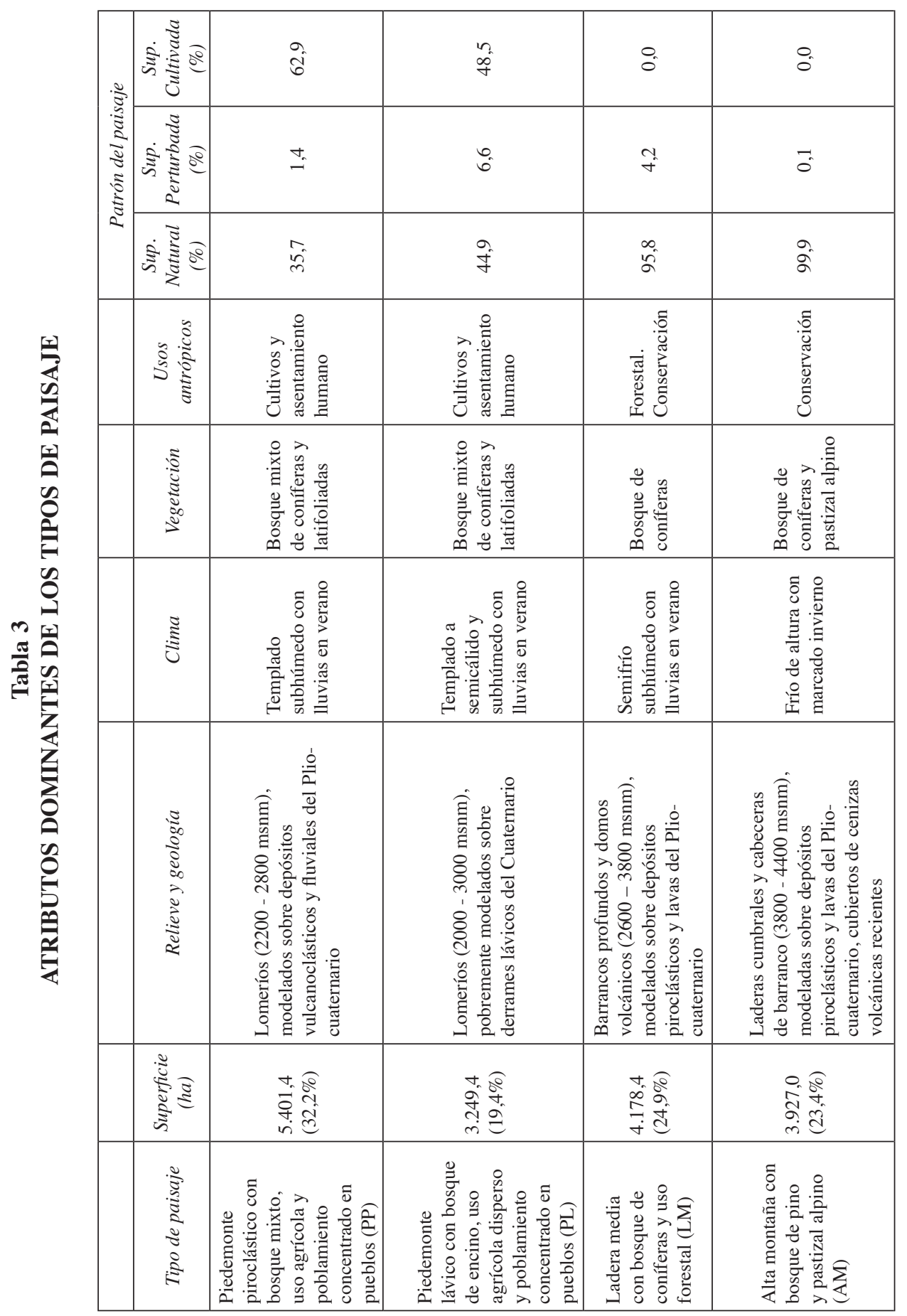


mente superior en la alta montaña, el piedemonte lávico y el piedemonte piroclástico que en la ladera media. Este índice caracteriza la penetración antropogénica de paisajes desde un punto de vista geométrico (Jaeger, 2000).

Finalmente, la representatividad / singularidad de estos paisajes es especialmente importante en la ladera media, que mostró el mayor valor de idoneidad desde la perspectiva de este estudio, mientras que la alta montaña tuvo la puntuación más baja. En conjunto, estos datos muestran una calidad relativamente homogénea entre los diferentes tipos de paisaje, aunque el patrón de paisaje de la ladera media se destaca como el tipo menos fragmentado y con la mayor representatividad.

Tabla 4

VALORES CALCULADOS PARA LOS ÍNDICES DE CALIDAD DE LOS TIPOS DE PAISAJE

\begin{tabular}{cccc}
\hline Tipo de Paisaje & $\begin{array}{c}\text { Diversidad Paisajística } \\
\text { (Índice de Simpson) }\end{array}$ & $\begin{array}{c}\text { Fragmentación } \\
\text { (Índice de División) }\end{array}$ & $\begin{array}{c}\text { Representatividad / } \\
\text { Singularidad } \\
\text { (Índice R/S). }\end{array}$ \\
\hline Piedemonte piroclástico & 0,67 & 3,06 & 1,27 \\
Piedemonte lávico & 0,71 & 3,52 & 1,40 \\
Ladera media & 0,65 & 2,93 & 1,96 \\
Alta montaña & 0,74 & 3,89 & 1,19 \\
\hline
\end{tabular}

\subsection{Inventario y valoración de los atractivos turísticos}

El inventario realizado permitió identificar 29 atractivos turísticos diferentes, cuya calidad fue evaluada mediante un conjunto de 29 indicadores ( 9 indicadores de calidad paisajística, 14 indicadores ambientales y 6 indicadores educativo/académicos). Estos, a su vez, evaluaron tres componentes claves del paisaje, a saber, los componentes bióticos, geomorfológicos y culturales (Tabla 2).

Los arenales del Popocatépetl fueron el atractivo turístico con mayor puntuación $(C a t=20,38)$, mientras que 16 atractivos turísticos $(55,2 \%$ del total $)$ fueron clasificados con valores medios y $12(41,4 \%)$ con valores bajos. Los indicadores ambientales que más contribuyeron al Cat fueron los relacionados con la vegetación $(=4,90)$, seguidos por los indicadores de relieve $(=3,60)$ y culturales $(=2,43)$. Esto resalta el predominio de los atributos naturales sobre los culturales - sin negar el valor de estos- y de los atractivos de calidad media (Cat entre 9,67 y 19,35) sobre los atractivos de calidad baja (Cat menor de 9,67) o alta (Cat entre 19,36 y 29,00) (Tabla 5). En cuanto al tipo de uso que se podría dar a los distintos atractivos, se observó una amplia gama de posibilidades para el ecoturismo, el turismo de aventura y el turismo rural, determinadas por las características intrínsecas (naturales o culturales) de cada atractivo. Cabe destacar que un atractivo turístico en sí puede servir a más de un tipo de uso, lo que aumenta su potencial. 


\section{Tabla 5}

INVENTARIO Y EVALUACIÓN DE LOS ATRACTIVOS TURÍSTICOS. $V b=$ Valor biótico; $V g=$ Valor geomorfológico; $V c=$ Valor cultural; $C a t=$ Calidad del atractivo turístico (Baja, Media, Alta)

\begin{tabular}{|c|c|c|c|c|c|c|c|c|c|}
\hline \multirow[b]{2}{*}{ No. } & \multirow[b]{2}{*}{ Nombre del atractivo } & \multirow[b]{2}{*}{$V b$} & \multirow[b]{2}{*}{$V g$} & \multirow[b]{2}{*}{$V c$} & \multirow[b]{2}{*}{ Cat } & \multirow[b]{2}{*}{ Calidad } & \multicolumn{3}{|c|}{ Uso potencial } \\
\hline & & & & & & & $\begin{array}{l}\text { Eco- } \\
\text { turismo }\end{array}$ & $\begin{array}{c}\text { Turismo } \\
\text { de } \\
\text { aventura }\end{array}$ & $\begin{array}{l}\text { Turismo } \\
\text { rural }\end{array}$ \\
\hline 1 & El Cedral 1 & 2,01 & 2,42 & 3,04 & 7,47 & Baja & $\mathrm{X}$ & & $\mathrm{X}$ \\
\hline 2 & Paraje Chilpayo & 2,82 & 5,28 & 2,23 & 10,33 & Media & $\mathrm{X}$ & & $X$ \\
\hline 3 & Paraje Chiltepe & 6,84 & 3,52 & 0,81 & 11,17 & Media & $\mathrm{X}$ & $\mathrm{X}$ & \\
\hline 4 & Paraje Calpisca & 3,22 & 1,76 & 3,04 & 8,02 & Baja & $\mathrm{X}$ & $\mathrm{X}$ & \\
\hline 5 & $\begin{array}{l}\text { Arenales del } \\
\text { Popocatépetl }\end{array}$ & 9,26 & 7,48 & 3,65 & 20,38 & Alta & $X$ & $X$ & \\
\hline 6 & Mirador arenales & 8,86 & 5,28 & 2,84 & 16,97 & Media & $\mathrm{X}$ & & \\
\hline 7 & Paraje Joya Redonda & 8,86 & 4,18 & 1,42 & 14,45 & Media & $\mathrm{X}$ & $\mathrm{X}$ & \\
\hline 8 & Paraje Metate Quebrado & 8,86 & 3,96 & 1,62 & 14,44 & Media & $\mathrm{X}$ & $\mathrm{X}$ & \\
\hline 9 & Paraje Chocoquiagua & 3,62 & 1,98 & 1,62 & 7,22 & Baja & $\mathrm{X}$ & $\mathrm{X}$ & \\
\hline 10 & $\begin{array}{l}\text { Domo volcánico } \\
\text { Tecamacapa }\end{array}$ & 4,03 & 4,18 & 2,43 & 10,64 & Media & $\mathrm{X}$ & $\mathrm{X}$ & $\mathrm{X}$ \\
\hline 11 & $\begin{array}{l}\text { Templo San Juan } \\
\text { Evangelista }\end{array}$ & 0,40 & 1,10 & 4,66 & 6,16 & Baja & & & $\mathrm{X}$ \\
\hline 12 & El Cedral 2 & 4,43 & 2,42 & 2,43 & 9,28 & Baja & $\mathrm{X}$ & $\mathrm{X}$ & $X$ \\
\hline 13 & Capilla San Juan Bautista & 0,81 & 2,86 & 6,08 & 9,74 & Media & & & \\
\hline 14 & Exhacienda Guadalupe & 0,40 & 1,10 & 4,46 & 5,96 & Baja & $\mathrm{X}$ & & \\
\hline 15 & Exhacienda Yautla & 0,40 & 1,76 & 2,43 & 4,59 & Media & $\mathrm{X}$ & & \\
\hline 16 & $\begin{array}{l}\text { Parroquia San Miguel } \\
\text { Arcángel }\end{array}$ & 0,40 & 2,86 & 6,08 & 9,34 & Baja & & & $\mathrm{X}$ \\
\hline 17 & Ruta del agua 1 & 3,22 & 2,64 & 1,22 & 7,08 & Baja & $\mathrm{X}$ & $\mathrm{X}$ & $\mathrm{X}$ \\
\hline 18 & Ruta del agua 2 & 7,65 & 4,40 & 1,42 & 13,47 & Media & $\mathrm{X}$ & $\mathrm{X}$ & \\
\hline 19 & Ruta del agua 3 & 8,45 & 4,84 & 0,41 & 13,70 & Media & $\mathrm{X}$ & $\mathrm{X}$ & \\
\hline 20 & $\begin{array}{l}\text { Ruta poblado Atlautla- } \\
\text { campo }\end{array}$ & 2,01 & 3,52 & 1,42 & 6,95 & Baja & $\mathrm{X}$ & $\mathrm{X}$ & $\mathrm{X}$ \\
\hline 21 & Ruta ciclista familiar & 5,64 & 4,84 & 1,42 & 11,89 & Media & $\mathrm{X}$ & & $\mathrm{X}$ \\
\hline 22 & Ruta ciclista de montaña & 8,45 & 4,62 & 1,42 & 14,49 & Media & $\mathrm{X}$ & & $\mathrm{X}$ \\
\hline 23 & Ruta mariposa monarca & 9,26 & 4,84 & 1,82 & 15,92 & Media & $\mathrm{X}$ & $\mathrm{X}$ & \\
\hline 24 & Paraje Mictlanco & 8,86 & 4,84 & 2,03 & 15,72 & Media & $\mathrm{X}$ & & $\mathrm{X}$ \\
\hline 25 & Árboles de navidad & 4,03 & 1,10 & 0,81 & 5,94 & Baja & & & $\mathrm{X}$ \\
\hline 26 & $\begin{array}{l}\text { Ruta al centro ceremonial } \\
\text { Xochiquia }\end{array}$ & 8,86 & 6,82 & 2,03 & 17,70 & Media & $\mathrm{X}$ & $\mathrm{X}$ & \\
\hline 27 & $\begin{array}{l}\text { Centro ceremonial } \\
\text { Xochiquia }\end{array}$ & 6,44 & 4,84 & 2,03 & 13,31 & Media & & & $\mathrm{X}$ \\
\hline 28 & Apiarios & 2,82 & 2,64 & 1,01 & 6,47 & Baja & $\mathrm{X}$ & & $X$ \\
\hline \multirow[t]{3}{*}{29} & Colonia Popo Park & 1,21 & 2,20 & 4,66 & 8,07 & Baja & & & $X$ \\
\hline & Máximo posible & 9,67 & 9,67 & 9,67 & 29,00 & & & & \\
\hline & Promedio & 4,90 & 3,60 & 2,43 & 10,93 & & & & \\
\hline
\end{tabular}




\subsection{Potencial turístico del paisaje}

La Figura 1 muestra la distribución de los atractivos turísticos en los diferentes tipos de paisaje. Como se observa, el piedemonte piroclástico incluye 13 atractivos turísticos $($ Cat acumulado $=112,60)$; la montaña alta, cuatro atractivos $(C a t$ acumulado $=68,36)$; el piedemonte lávico, siete (Cat acumulado $=68,13$ ); y la ladera media, cinco (Cat acumulado $=67,75$ ). Por otro lado, en cuanto a la calidad de los atractivos, la mayor contribución al Cat del piedemonte piroclástico $(63,94)$ proviene de los atractivos de Cat baja, complementado por atractivos de Cat media $(48,66)$. El piedemonte lávico muestra el patrón opuesto, es decir, los atractivos de Cat media (46.76) hacen una contribución más alta en relación con los de Cat baja $(21,37)$. Este comportamiento es particularmente pronunciado en la ladera media, cuya Cat proviene principalmente de atractivos con Cat media $(60,53)$, con una pequeña contribución de los de Cat baja $(7,22)$. La alta montaña contiene al único atractivo de Cat alta $(20,38)$, y los atractivos de Cat baja están ausentes, sin embargo, como es también el caso del piedemonte lávico y la ladera media, su valor de Cat proviene principalmente de atractivos de Cat media $(47,98)$. La relación entre el Cat acumulado, la calidad de los atractivos y los tipos de paisaje se muestra en la Figura 2.

\section{Figura 1 \\ TIPOS DE PAISAJE Y ATRACTIVOS TURÍSTICOS DEL MUNICIPIO DE ATLAUTLA, VERTIENTE OCCIDENTAL DEL VOLCÁN POPOCATÉPETL, CENTRO DE MÉXICO}

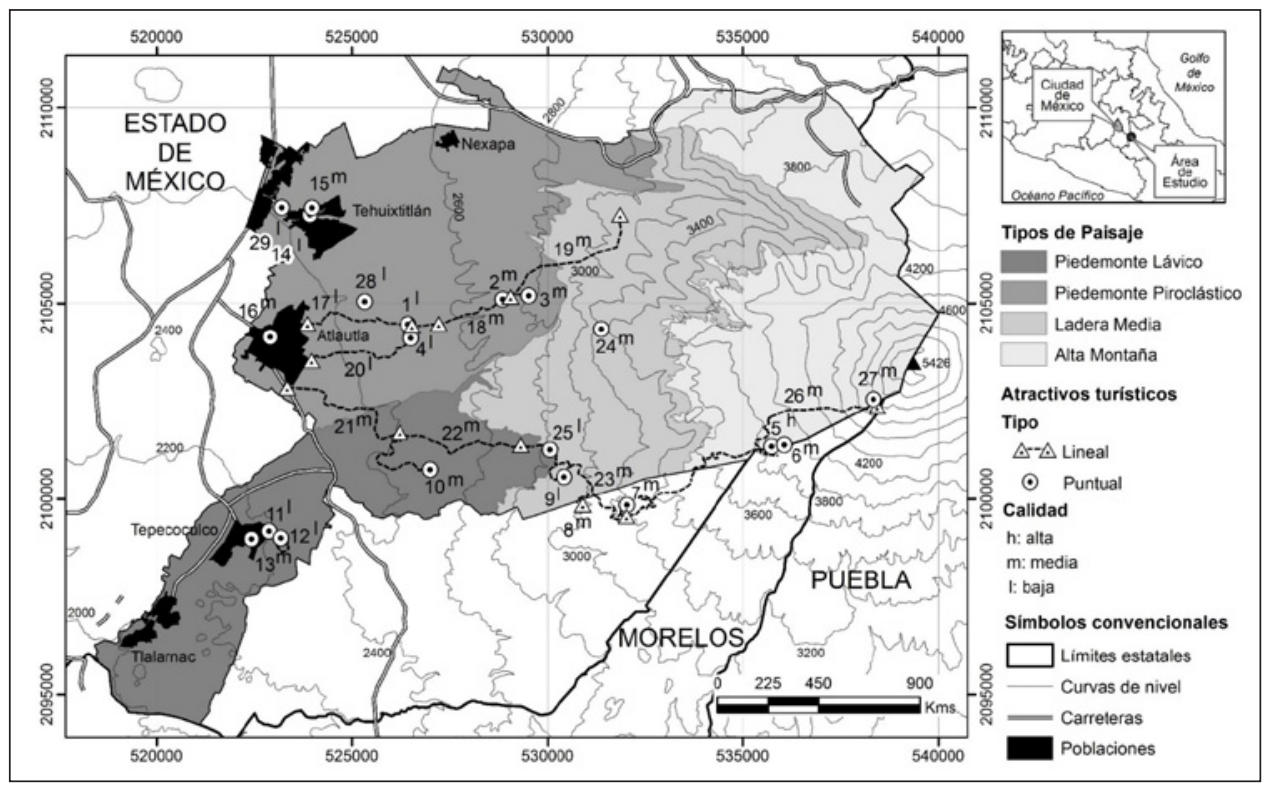


Estos resultados sugieren que el potencial turístico del municipio de Atlautla se sustenta en patrones paisajísticos de calidad suficiente, caracterizados por una alta diversidad, un buen estado de conservación y la presencia significativa de elementos educativos, complementados por la presencia de atractivos turísticos que otorgan un valor agregado a los tipos de paisaje, tanto en términos de frecuencia como de calidad. De hecho, fue la frecuencia de los atractivos lo que confiere al piedemonte piroclástico un mayor valor agregado que los otros tres tipos de paisajes, en los que la calidad de los atractivos fue más importante que su frecuencia (Figura 2).

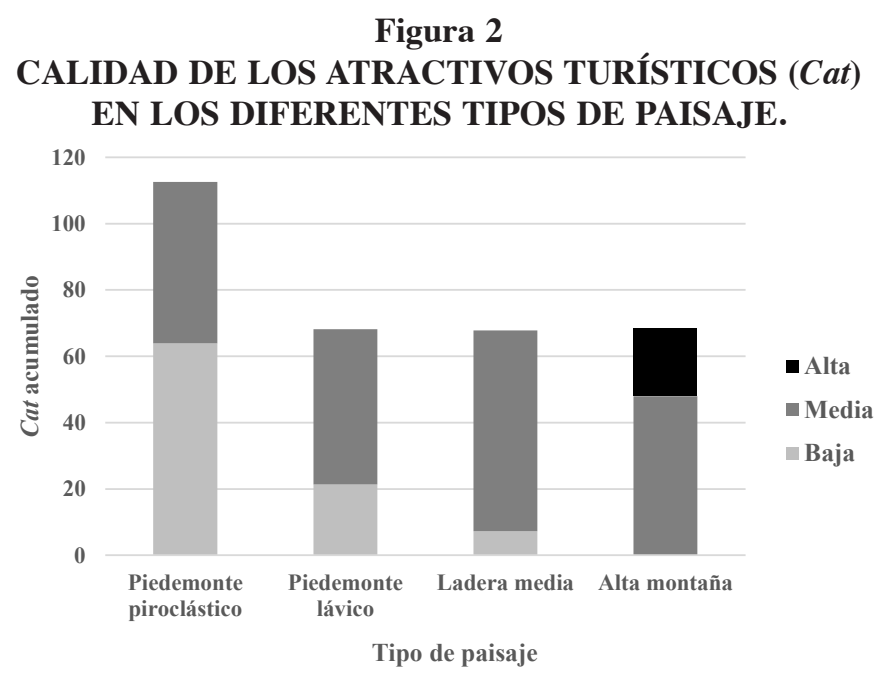

Cat acumulado = suma del $\boldsymbol{C a t}$ de los atractivos turísticos de una cierta calidad paisajística (alta, media o baja) en el correspondiente tipo de paisaje

\section{DISCUSIÓN}

\subsection{Importancia de la evaluación del potencial del paisaje como recurso turístico en el medio rural}

Este documento propone un enfoque original para evaluar el potencial turístico de los paisajes, entendidos desde planteamientos de reconocimiento geosistémico, en áreas de marcado carácter rural, tradicionalmente dedicadas a las actividades agropecuarias, aunque actualmente en declive, donde se conoce la existencia de un amplio capital natural y cultural, y un interés de las comunidades locales para el desarrollo del turismo. En ese sentido, el carácter visual del paisaje lo convierte en una herramienta útil para vincular al sector académico con las perspectivas e intereses de las comunidades locales, proporcionando nuevos enfoques a las actuales tendencias científicas e institucionales que promueven la importancia del paisaje como elemento de planificación y gestión (Council of Europe, 2000, OCDE, 2003, Barbini, 2005; Mata, 2011). 
Por otra parte, cabe destacar la naturaleza aplicada de este estudio, principalmente porque la mayoría de las propuestas representan enfoques parciales: a) como un marco general de integración que comprende un conjunto de atracciones (usualmente como lugares del patrimonio cultural) (Stange et al., 2013), o b) como características geográficas relevantes (a menudo impulsadas por las condiciones climáticas) de los atractivos turísticos (Kane, 1981; Milheiro et al., 2013). En este caso, la calidad de los atractivos turísticos se integró con la calidad de los tipos paisajísticos, como valor añadido que aumenta el potencial turístico del paisaje. La consideración de tres criterios de evaluación del paisaje, ampliamente aceptados (escénicos, ambientales y culturales), es clave para la aplicación del método a otras zonas rurales diferentes e incluso contrastantes. Los resultados obtenidos para el municipio de Atlautla permiten afirmar que el paisaje es un recurso que armoniza las condiciones naturales y culturales del territorio, algunas de las cuales pueden ser evaluadas como recursos para el desarrollo del turismo (Escriche, 1999; Zuluaga, 2006; Dosso, 2011; Picazo, 2012; Carneiro et al., 2015; Cebrián y García, 2016).

\subsection{Sobre la selección de indicadores para determinar el potencial turístico del paisaje}

Desde el punto de vista teórico-metodológico, este estudio se basó en el diseño y aplicación de indicadores de calidad para el diagnóstico del potencial turístico del paisaje en zonas rurales. Como complemento a los enfoques que consideran al paisaje desde una perspectiva estética o visual (Zuluaga, 2006; Knudsen et al., 2013; Kalivoda et al., 2014), se ha sugerido un conjunto de indicadores mensurables y objetivos que hacen referencia a los distintos componentes físico, biótico y cultural para proporcionar una visión integrada del conjunto de recursos disponibles en el paisaje (Cawley y Gilmor, 2008). Esto ayudó a construir una evaluación altamente explicativa, sistemática e integral, con un mayor margen para objetivos prácticos de uso (Serrano, 2008; Nogué et al., 2009), y más adecuada para promover el turismo rural alternativo, sostenible e integrado (Butler, 1999; Kenafsey, 2001; Salinas y La O, 2006; Cawley y Gillmor, 2008; Prat Forga y Cànoves Valiente, 2014).

Nuestra experiencia en el municipio de Atlautla confirma los resultados de estudios previos, en los que la identificación y evaluación de indicadores del paisaje en el campo y con el apoyo de la comunidad, facilita el compromiso de los actores locales en el diseño de proyectos de desarrollo turístico, lo cual es uno de los principales requerimientos para la implementación y el desarrollo exitoso de estas modalidades económicas (Barbini, 2005; Picazo, 2012; Hernández el al., 2012; Méndez et al., 2016).

\subsection{Sobre la integración de los atractivos turísticos en los tipos de paisaje}

El área de estudio presenta una amplia diversidad de paisajes, lo cual encaja de manera consistente con las nuevas modalidades del denominado turismo alternativo (Berry y Ladkin, 1997; Lane y Kastenholz, 2015). Efectivamente, de los tres tipos de turismo alternativo reconocidos: ecoturismo, turismo de aventura y turismo rural (Alcalá y López, 2017), en Atlautla sería posible centrar la oferta turística en torno a los dos primeros, básicamente a través de la implementación de actividades recreativas en los diversos atractivos 
turísticos ubicados en las áreas forestales y de montaña (Beedie y Hudson, 2003; Waitt et al., 2003). Por su parte, el turismo rural, aunque menos desarrollado en la región, podría basarse en el aprovechamiento de: a) las antiguas construcciones históricas del tiempo de la colonia española, b) los centros místico-religiosos de tradición prehispánica, ubicados sobre las laderas medias y altas del volcán, c) las extensas áreas de cultivo aún poco valoradas por la población urbana del centro del país, e incluso d) algunos productos de tipo gastronómico, que aunque no son exclusivos del municipio, son regularmente requeridos por el turismo nacional (Torres, 2003; Willson et al., 2013).

Particularmente, se identificaron diversos atractivos turísticos que pueden ser el objeto central de los proyectos detonadores de la actividad turística. Estos atractivos son básicamente aquellos de valor de calidad media, a partir de los cuales se puede impulsar el aprovechamiento de otros atractivos de menor calidad (Cat), integrándose todos ellos mediante los tipos de paisaje de alta calidad. Así, los paisajes y los atractivos de calidad turística le confieren al territorio de estudio un mayor potencial, por ejemplo, para el diseño de rutas turísticas - u otro tipo de productos- que favorezcan el desarrollo integral de la actividad turística, una mayor capacidad para satisfacer, desde el punto de vista de la demanda social, la micro-movilidad de un extenso mercado (Lane y Kastenholz, 2015) -en este caso concentrado en la Ciudad de México-, así como ampliar los beneficios de la actividad a un sector más amplio de la sociedad local.

Los habitantes de Atlautla tienen una clara percepción del potencial que los recursos paisajísticos aportan al desarrollo del turismo. No obstante, ellos reconocen que el municipio presenta sensibles carencias en algunos aspectos que son básicos para una real posibilidad de crecimiento (Méndez et al., 2016). Efectivamente, el éxito del turismo como actividad complementaria para paliar la pobreza en el mundo rural, requiere soluciones integrales que consideren la atención a otros problemas vinculados con la construcción de infraestructura básica - que usualmente debería ser provista por el sector público (Lane y Kastenholz, 2015) -, el empoderamiento de los usos tradicionales, la solución de los conflictos relacionados con la propiedad de la tierra, la organización del comercio y la emigración de la población joven.

\section{CONCLUSIONES}

Durante décadas, el turismo ha sido considerado como una actividad clave para la reestructuración económica de las zonas rurales en muchos países, sin embargo, muchos enfoques para el desarrollo de esta actividad han considerado el potencial turístico sin incluir una evaluación objetiva del paisaje. En este caso, la evaluación del paisaje en términos de calidad paisajística, ambiental y cultural se llevó a cabo mediante un enfoque pragmático para responder a la necesidad de identificar los factores geosistémicos y de percepción que determinan la calidad del paisaje desde el punto de vista turístico. Asimismo, se consideró como criterio central de la ponderación, el "valor agregado" asociado a la frecuencia y calidad de los atractivos, que desempeñan un papel claramente definido como productos que se pueden ofrecer de inmediato a los visitantes, abarcando una amplia gama de posibilidades dentro del llamado turismo alternativo (ecoturismo, turismo de aventura y turismo rural). 
Se espera que este enfoque metodológico, basado en la combinación de criterios aplicados a dos niveles de análisis de los recursos turísticos (paisajísticos y de atractivos), pueda ser empleado en otras comunidades rurales de diferente naturaleza, como herramienta de apoyo a la adecuada planificación de los proyectos locales durante las etapas de diagnóstico. Además, el método aquí propuesto puede complementar otros enfoques, en particular los que apoyan la viabilidad social del turismo, basados en la participación directa de las comunidades locales como elemento central para el desarrollo de proyectos de turismo sostenible.

La evaluación del potencial turístico del paisaje mediante la integración de un conjunto de indicadores objetivos constituye un buen ejercicio de evaluación integral del territorio que puede contribuir al desarrollo económico de las zonas rurales fundamentado en un turismo rural integrado y sostenible, capaz de respetar y asegurar las principales características de las áreas involucradas.

\section{BIBLIOGRAFÍA}

AITCHISON, C., MACLEOD, N. y SHAW, S. (2001): Leisure and Tourism Landscapes: Social and Cultural Geographies. London, Routledge.

ALCALÁ, B. y LÓPEZ, A. (2017): «Zonas con potencial agroturístico en la región citrícola de Nuevo León, México: un análisis a partir del algebra de mapas», Cuadernos de Turismo, $\mathrm{n}^{\circ} 39$, pp. 17-40.

BARBINI, B. (2005): «Viabilidad social para el desarrollo turístico en centros urbanos bonaerenses», Aportes y transferencias, vol. 9, $\mathrm{n}^{\circ} 2$, pp. 148-158.

BEEDIE, P. y HUDSON, S. (2003): «Emergence of mountain-based adventure tourism», Annals of Tourism Research, vol. 30, n 3, pp. 625-643.

BERRY, S. y LADKIN, A. (1997): «Sustainable tourism: A regional perspective», Tourism Management, $\mathrm{n}^{\circ} 18$, pp. 433-440.

BERTRAND, C. y BERTRAND, G. (2006): Geografía del medio ambiente: el sistema GTP: geosistema, territorio y paisaje. Universidad de Granada.

BUTLER, R. (1999): «Sustainable Tourism: A State-of-the-Art Review», Tourism Geographies, $\mathrm{n}^{\circ} 1$, pp. 7-25.

CARNEIRO, M.J., LIMA, J. y SILVA, A.L. (2015): «Landscape and the rural tourism experience: identifying key elements, addressing potential, and implications for the future», Journal of Sustainable Tourism, vol. 23 (8-9), pp. 1.217-1.235.

CEBRIÁN, F. y GARCÍA, C. (2016): «Uso y gestión del paisaje para la actividad turística en el medio rural: aproximación teórica y empírica en el sureste de Castilla-La Mancha (Albacete)», Boletín de la Asociación de Geógrafos Españoles, nº 72, pp. 381-407.

COUNCIL OF EUROPE (2000): European Landscape Convention. European Treaty Series, No. 176. Strasbourg.

CAWLEY, M. y GILLMOR, D.A. (2008): «Integrated rural tourism: Concepts and Practice». Annals of Tourism Research, Vol. 35, n 2, pp. 316-337.

DOSSO, R.H. (2011): «Turismo y paisaje: pluralidad conceptual, versatilidad interpretativa y responsabilidad interdisciplinar», Aportes y transferencias. Tiempo Libre. Turismo y Recreación, año 15, vol. II, pp. 15-54. 
ESCRICHE, M.M. (1999): «Medio físico y turismo rural: una aportación para los informadores turísticos». Cuadernos de Turismo, n 3, pp. 93-114.

GARCÍA-ROMERO, A. (2002): «El paisaje: una herramienta para el estudio detallado del territorio», Kuxulkab', vol. VII, n 14, pp. 22-33.

GORDON, T. (1994): «The Delphi Method», in Glen, J.C. y Gordon, T. J. (Eds.) Futures Research Methodology, American Council for The United Nation University, Millennium Project, pp. 1-33.

HERNÁNDEZ, I., SALINAS, E. y PÉREZ, E. (2012): «Consideraciones metodológicas para el desarrollo del ecoturismo a partir de las relaciones inter y transectoriales. Estudio de caso: Parque Nacional Viñales, Cuba», Grand Tour, n 5, pp. 8-26.

INEGI (1983): Cartas de uso de suelo y vegetación. Escala 1.50 000. E14B41 Amecameca, E14B42 huejotzingo, E14B51 Cuautla. Ciudad de México, Instituto Nacional de Estadística, Geografía e Informática.

INEGI (1996): Cartas topográficas. Escala 1.50 000. E14B42 Huejotzingo, E14B41 Amecameca, E14B51 Cuautla. Ciudad de México, Instituto Nacional de Estadística, Geografía e Informática.

INEGI (2009): Prontuario de información geográfica municipal de los Estados Unidos Mexicanos. Atlautla, México. Clave geoestadística 15015. Ciudad de México, Instituto Nacional de Estadística, Geografía e Informática.

JAEGER, J.A.G. (2000): «Landscape division, splitting index, and effective mesh size: new measures of landscape fragmentation», Landscape Ecology, $\mathrm{n}^{\circ} 15$, pp. 115-130.

JEREZ, O. y SERRANO DE LA CRUZ, M. A. (2016): «El interés didáctico de los paisajes alterados. La Reserva de la Biosfera de La Mancha Húmeda (España) como ejemplo de estudio», Contexto \& Educaçao, v. 31, no 99, pp. 52-80.

KALIVODA, O., VOJAR, J., SKRIVANOVÁ, Z. y ZAHRADNÍK, D. (2014): «Consensus in landscape preference judgments: The effects of landscape visual aesthetic quality and respondents' characteristics», Journal of Environmental Management, $\mathrm{n}^{\circ}$ 137, pp. 36-44.

KANE, P.S. (1981): «Assessing landscape attractiveness: a comparative test of two new method», Applied Geography, vol. 1, n 2, pp. 77-96.

KENAFSEY, M. (2001): «Rural cultural economy: tourism and social relations», Annals of Tourism Research, vol. 28, no 3 , pp. 762-783.

KNUDSEN, D.C., GREER, C.E., METRO-ROLAND, M. y SOPER, A.K. (Eds.) (2008): Landscape, Tourism and Meaning. Aldershot, Hampshire, UK, Ashgate.

KNUDSEN, D.C., METRO-ROLAND, M.M. y RICKLY-BOYD, J.M. (2013): «Landscape studies and tourism research», in Howard, P., Thompson, I. y Waterton, E. (Eds.) The Routledge Companion to Landscape Studies, New York, Routledge, pp. 286-295.

LANE, B. y KASTENHOLZ, E. (2015): «Rural tourism: the evolution of practice and research approaches - towards a new generation concept?», Journal of Sustainable Tourism, vol. 23, no 8-9, pp. 1133-1156.

MATA, R. (2011): «La gestión del paisaje», en Simancas, M. y CORTINA, A. (coords.) Retos y perspectivas de la gestión del paisaje en Canarias. Reflexiones en relación con el $10^{\circ}$ aniversario de la firma del Convenio Europeo del Paisaje. Tenerife, UIMP y Gobierno de Canarias, pp. 19-40. 
MATEO, J.M. (2008): Paisajes naturales, Geografía de los Paisajes, Primera Parte. La Habana, Universitaria.

MATEOS M.R. (2016): «Paisaje, patrimonio y turismo de surf: factores de atracción y motivación en el Parque Natural del Estrecho, España», Cuadernos de Turismo, ${ }^{\circ}$ 37, pp. 351-376.

MEARNS, K.F. (2012): «Lessons from the application of sustainability indicators to community-based ecoturism ventures in Southern Africa», African Journal of Business Management, vol. 6, $\mathrm{n}^{\circ}$ 26, pp. 7851-7860.

MÉNDEZ, A., GARCÍA-ROMERO, A., SERRANO DE LA CRUZ, M.A. y IBARRA, V. (2016): «Determinantes sociales de la viabilidad del turismo alternativo en Atlautla, una comunidad rural del centro de México», Investigaciones Geográficas. Boletín del Instituto de Geografía, UNAM, nº 90, pp. 119-135.

MENG, F. y UYSAL, M. (2008). «Effects of Gender Differences on Perceptions of Destination Attributes, Motivations and travel Values: An Examination of a Nature - based Resort Destination», Journal of Sustainable Tourism, vol. 16, n 4, pp. 445-466.

MIKULEC, J. y ANTOUŠKOVÁ, M. (2011): «Landscape and tourism potential in the protected landscape areas», Agricultural Economics Czech, n 6, pp. 272-278.

MILHEIRO, E., ALVES, J.E. y MARTINS, A. (2013): The rural heritage as a rural tourism. Towards a new rurality?, in De Carvalho, A, Ravaglia, L. y Lage, R. (coods.) Turismo no Espaço Rural. Alternativas sustentáveis para o desenvolvimento do meio rural, Conference: ESADR, VII Congresso da APDEA, V Congresso da SPER, I Encontro Lusófono em Economia, Sociologia, Ambiente e Desenvolvimento Rural, Universidade de Évora, pp. 2.829-2.918.

MUÑOZ, J. (1998): «Paisaje y geosistema. Una aproximación desde la Geografía Física», en Martínez de Pisón, E. (Dir.) Paisaje y Medio Ambiente, Valladolid, Fundación Duques de Soria y Universidad de Valladolid, pp. 45-55.

NIKODEMUS, O., BELL, S., GRINE, I. y LIEPINŠ, I. (2005): «The impact of economic, social and political factors on the landscape structure of the Vidzeme uplands in Latvia», Landscape and Urban Planning, vol. 70, $\mathrm{n}^{\circ} 1-2$, pp. 57-67.

NOGUÉ, J. (1992): «Turismo, percepción del paisaje y planificación del territorio», Estudios Turísticos, $\mathrm{n}^{\circ} 115$, pp. 45-54.

NOGUÉ, J., PUIGBERT, L. y BRETCHA, G. (Eds.) (2009): Indicadors de paisatge. Reptes i perspectives, Barcelona, Observatorio del Paisaje de Cataluña, Obra Social de Caixa Catalunya.

OECD (2003): OECD Environmental Indicators: Development, Measurement and Use, Paris, Organization for Economic Co-operation and Development.

PEET, R. (1974): «The measurement of species diversity», Annual Review of Ecology and Systematics, vol. 5, n 1, pp. 285-307.

PICAZO, H. (2012): «¿Por qué viajamos donde viajamos? Una Introducción a la estrecha relación entre Turismo y Paisaje», Revista Eubacteria, n 29 , pp. 1-5.

POWELL, G., BARBORAK, J., y RODRÍGUEZ, M. (2000): «Assessing representativeness of protected natural areas in Costa Rica for conserving biodiversity: a preliminary gap analysis», Biological Conservation, vol. 93, nº 1, pp. 35-41. 
PRAT FORGA, J.M., y CÀNOVES VALIENTE, G. (2014): «Integrated cultural tourism: new experiences in mountain areas», Tourisms. An International Multidisciplinary Journal of Tourism, vol. 9, $\mathrm{n}^{\circ}$ 2, pp. 15-32.

SALINAS, E. y La O, J.A. (2006): «Turismo y sustentabilidad: de la teoría a la práctica en Cuba», Cuadernos de Turismo, n 17, pp. 203-223.

SALINAS, E., NAVARRO, E., ECHARRI, M. y LA O, J.A. (2008): «Metodología para la evaluación de la sustentabilidad territorial: el uso de indicadores en destinos turísticos de Cuba», Boletín de la Real Sociedad Geográfica, n 144, pp. 77-102.

SANTOS-PAVÓN, E., FERNÁNDEZ-TABALES, A. y MUÑOZ-YULES, O. (2016): «La incorporación del paisaje a la planificación turística. Análisis de la estrategia de turismo sostenible de Andalucía», Cuadernos de Turismo, n 37, pp. 175-202.

SERRANO, D. (2008): «Ensayo metodológico para la valoración estética del paisaje. Aplicación en Muntanyes D’Ordal, Barcelona», Geographicalia, n 54, pp. 99-112.

SIMPSON, E.H. (1949): «Measurement of Diversity», Nature, n 163, p. 688.

SMITH S., SILVA J.F. y FARIÑAS M.R. (2008): «Diversidad, estabilidad y dinámica del paisaje en comunidades de sabana», Ecotrópicos, vol. 21, n 2, pp. 89-96.

STANGE, J., BROWN, D. HILBRUNER, R., y HAWKINS D.E. (2013): Tourism destination management. Achieving sustainable and competitive results, Washington, D.C., United States Agency for International Development (USAID).

TARROJA, A. (2004): «Paisaje y gestión del territorio: transformaciones territoriales y valoración social del paisaje», Monográfico sobre paisaje y gestión del territorio, Madrid, Colegio de Geógrafos, pp. 1-8.

TORRES, R. (2003). «Linkages between tourism and agriculture in México», Annals of Tourism Research, vol. 30, $\mathrm{n}^{\circ} 3$, pp. 546-566.

WAITT, G., LANE, R., y HEAD L. (2003): «The boundaries of nature tourism», Annals of Tourism Research, vol. 30, $\mathrm{n}^{\circ} 3$, pp. 523-545.

WILLSON, G.B., MCINTOSH, A.J. y ZAHRA, A.L. (2013): «Tourism and Spirituality: a Phenomenological Analysis», Annals of Tourism Research, $\mathrm{n}^{\circ}$ 42, pp. 150-168.

WLODARCZYK, B. (2009): «The landscapes of tourism space», Tourism, vol. 19, $\mathrm{n}^{\circ}$ 1-2, pp. 83-90.

ZULUAGA, P.A. (2006): «Una mirada al paisaje como recurso turístico», Revista Interamericana de Ambiente y Turismo, vol. 2, n 2, pp. 76-82. 